13 - ORIGINAL ARTICLE

TECHNICAL SKILL

\title{
Nitric oxide levels in cochlear fluids of guinea pig ${ }^{1}$
}

\author{
Fernando Kaoru Yonamine ${ }^{\mathrm{I}}$, Ektor Tsuneo Onishi ${ }^{\mathrm{II}}$, Margareth Gori Mouro ${ }^{\mathrm{III}}$, Elisa Mieko Suemitsu Higa ${ }^{\mathrm{IV}}$, Oswaldo Laércio \\ Mendonça Cruz ${ }^{\mathrm{v}}$
}

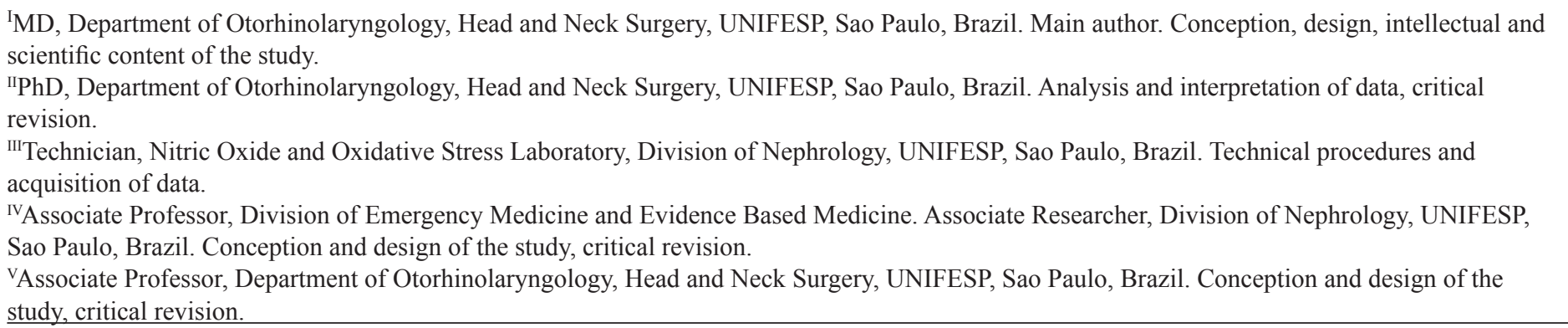

\section{ABSTRACT \\ PURPOSE: To describe a technique to obtain guinea pigs cochlear fluids and measure nitric oxide (NO) concentration.}

METHODS: Six guinea pigs were used and sacrificed. The cochlear fluids collected for measurement of NO, performed by chemiluminescence (NOA 280).

RESULTS: Through the chemiluminescence was possible to analyze the concentration of NO in cochlear fluids obtained. Average levels of nitric oxide from guinea pigs was $12.55 \mu \mathrm{M}$.

CONCLUSION: It is possible to obtain nitric oxide cochlear fluids, with this technique and nitric oxide concentration measure by chemiluminescence, a quantitative and more precise method.

Key words: Nitric Oxide. Cochlea. Guinea Pigs. 


\section{Introduction}

The search for an experimental model that provides a detailed and reliable study of the physiopathology of a wide range of human diseases is a constant challenge for scientists. The ideal model must reproduce these mechanisms, being at the same time, reproducible and easily executed. Low cost is another important characteristic for its feasibility worldwide.

In Otology, especially in the study of sensorineural hearing loss, experimental models are of utmost importance. Such models have been widely used and they resort to many technical resources and different species of animals.

Among all experimental animals, guinea pig is preferred due to its biological resistance in captivity. Apart from that, its anatomy favors an adequate accessibility to the middle and inner ear via bulla mastoidea, which is well developed in guinea pigs ${ }^{1,2}$

The main cause of sensory hearing loss is the injury in ciliated cells, and, the role of free radicals in producing cell lesion is gaining prominence for the evidences that lead to its participation in several pathological situations such as ototoxicity, hearing loss and cochlear ischemia ${ }^{3-5}$.

Most studies target the analysis of Nitric Oxide (NO), a free radical, produced by the action of nitric oxide synthase (NOS), its stable metabolites are nitrite and nitrate ${ }^{6}$. There are two main forms related to NOS: constitutive, whose activation depends on the calmodulin calcium complex, with two isomers called NOS I or neuronal NOS (nNOS) and NOSIII or endothelia NOS (eNOS) and a second one called inducible NOS (iNOS) or NOSII, whose activation is related to endotoxins and cytokines ${ }^{6-8}$.

Cochlear nitric oxide was demonstrated by Fessenden et al. ${ }^{9}$ through a histochemical technique based on NADPH diaphorase. This technique utilizes NOS capacity of reducing nitroblue tetrazolium into a dark blue insoluble compound that can be seen in histological samples. In this work NOS was present in the internal and external ciliated cells of the nerve endings, and, in guinea pigs' cochlear spiral ganglion cells. Despite the fact that NOS isoforms were not singled out, this was a very specific technique for nervous tissue ${ }^{10}$.

NO measurement in cochlear tissues can be done via indirect method (when its metabolites nitrite and nitrate are measured) or by direct ones (NO count). It can also have either qualitative or quantitative assessment.

Presently the difficulty that the majority of authors have to tackle with is how to perform NO measurement in a quantitative fashion and in an intra cochlear environment in order to better understand and study its participation in experimental models. The difficulty is to collect sufficient amounts of cochlear fluids from guinea pigs to enable NO counts. Therefore the development of a technique that allows the collection of an adequate amount of NO for ototoxicity experimental research would be very important and several other studies would take advantage of the use of this model.

\section{Methods}

Experiments were performed on six adult male guinea pig (Cavia porcellus), weighting around 500g, with positive Preyer's reflex ${ }^{11}$. They were maintained in cages, fed and provided with water, under controlled temperature and illumination. This experimental study was approved by the Ethic and Research Committee of the Federal University of Sao Paulo, protocol n. 0794/08.

Guinea pigs were submitted to intraperitoneal ketamine chloridrate anesthesia $(15 \mathrm{mg} / \mathrm{kg})$ and xilazine chloridrate $(3 \mathrm{mg} / \mathrm{kg})$. The animals were placed in lateral decubitus exposing the retro auricular region of one of the ears to infiltrate $2 \%$ lidocaine solution with vascular constrictor (Figure 1). Following the anesthesia an incision in the retro auricular region was performed with $n^{\circ} 15$ scalpel (Figure 2) and the dissection in planes with iris scissor allowed the exposition of bulla mastoidea (Figure 3). Bulla mastoidea was opened with a high speed motor and a $2 \mathrm{~mm}$ drill, under optical microscope (Figure 4), to meet the round window niche (Figure 5). The cochlear fluid was aspirated with a micropipette and an adapted insulin needle (Figure 6) by perforating the round window membrane (Figure 7). The fluid was kept in appropriate recipients under $0^{\circ} \mathrm{C}$ temperature. The same surgical procedure was performed on the other ear and the animals were sacrificed at the end of the experiment.

Despite the low negative pressure produced by the micropipette, its association with the manipulation of an insulin needle in the tympanic ramp presents a potential risk for membranous labyrinth membranes rupture. Therefore, the final aspirated product from the round window is probably a mixture of endolymph and perilymph, and hence named herein "cochlear fluids". 


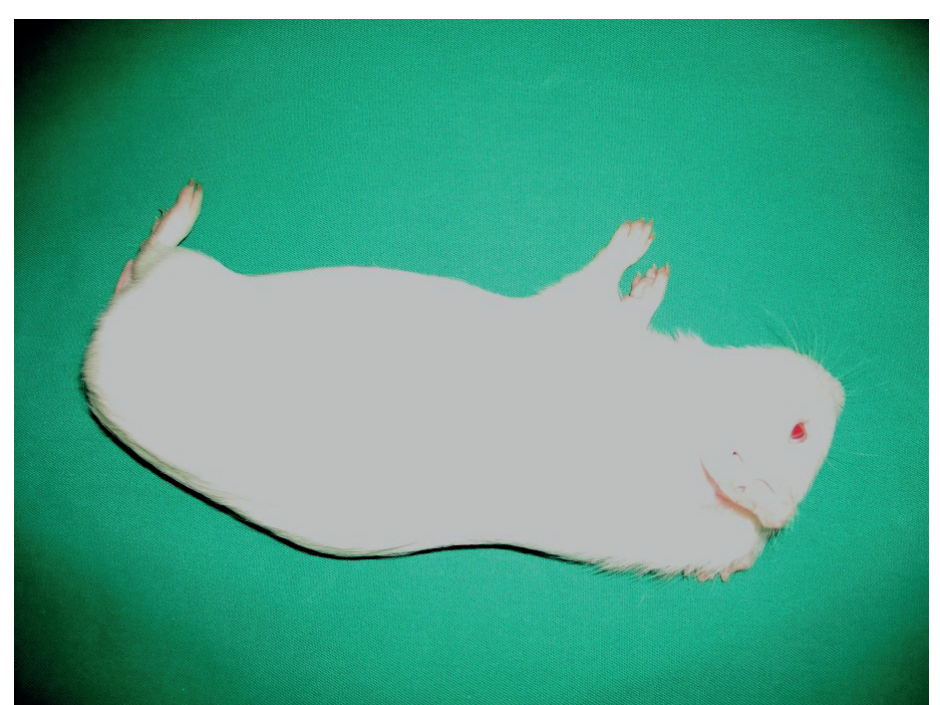

FIGURE 1 - Guinea pig in right lateral decubitus to approach the left ear.

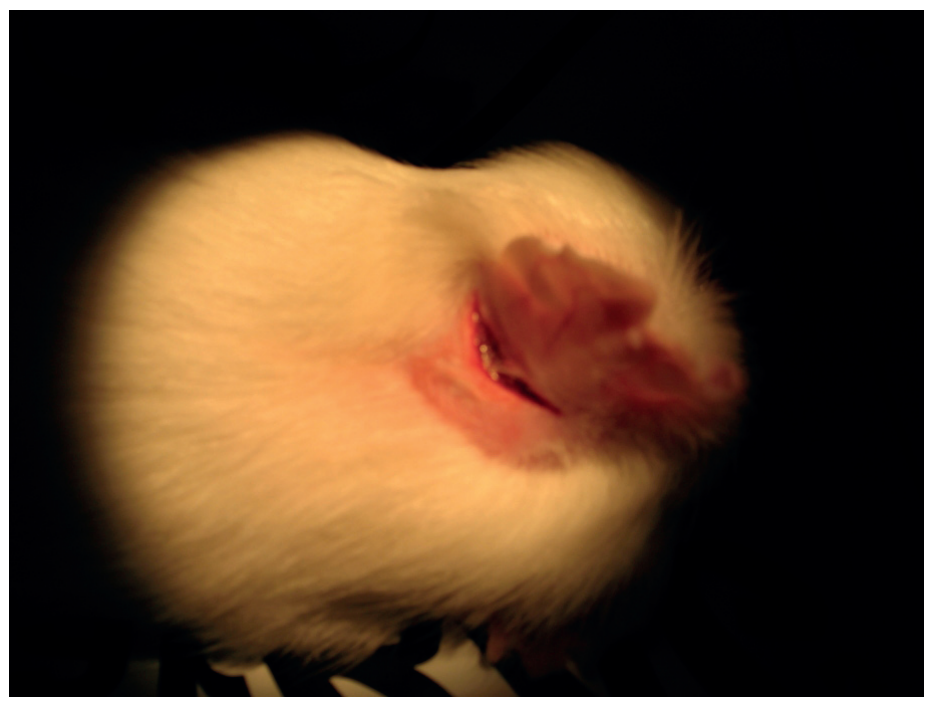

FIGURE 2 - Retro auricular incision of the skin.

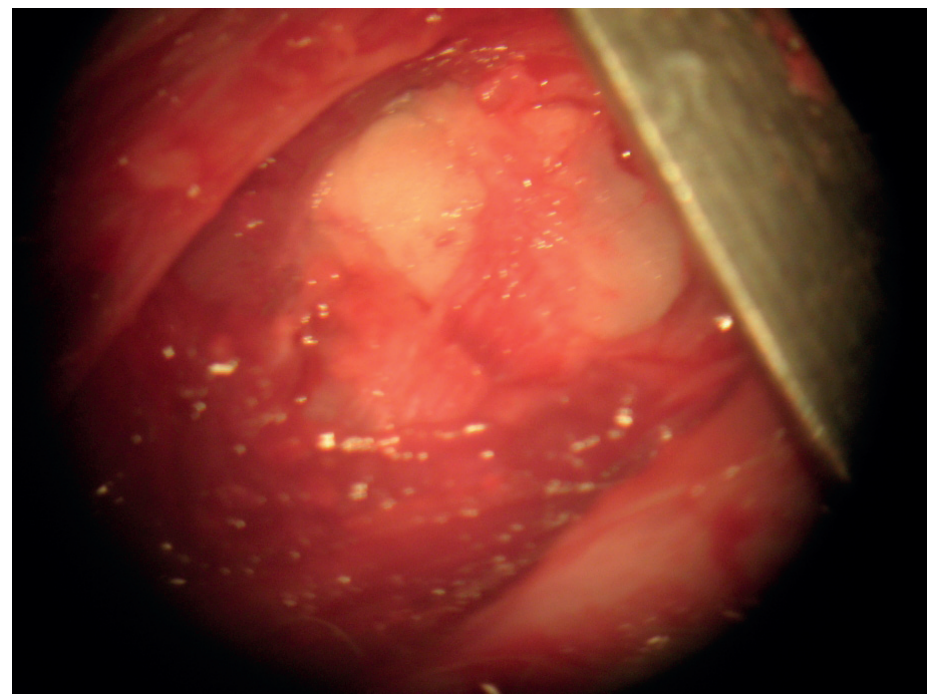

FIGURE 3 - Identification of bulla mastoidea and presentation with otostatic surgical retractor.

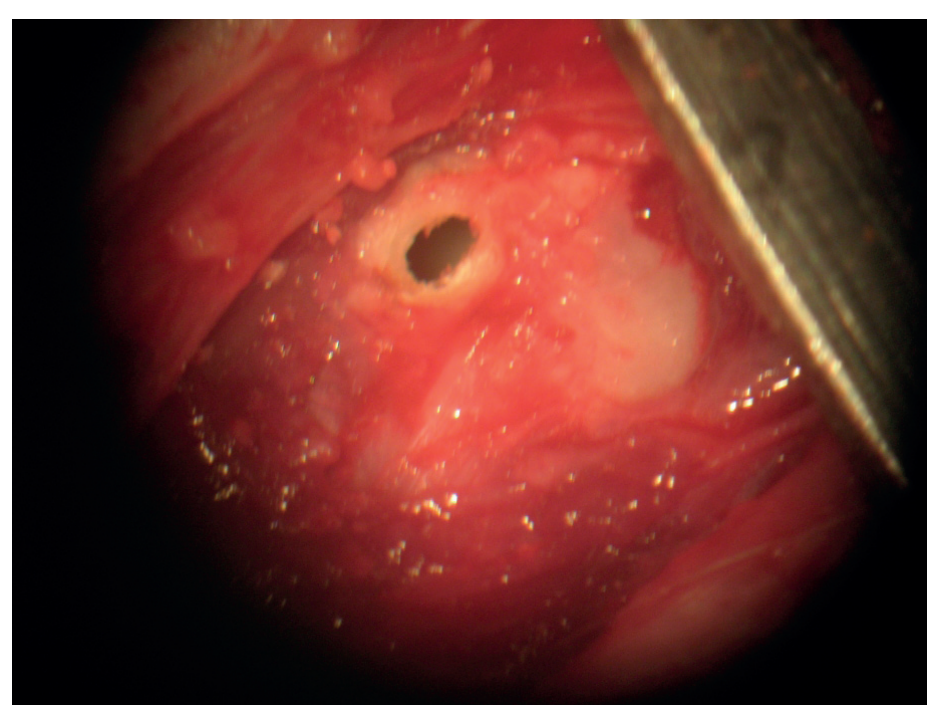

FIGURE 4 - Bulla mastoidea opening.

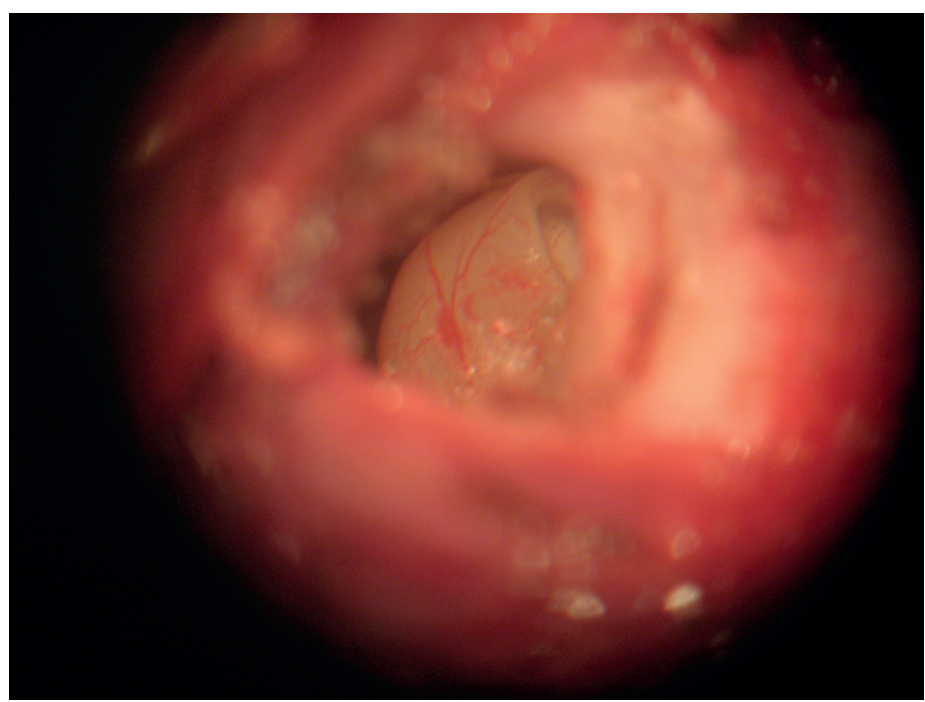

FIGURE 5 - Identification of round window niche.

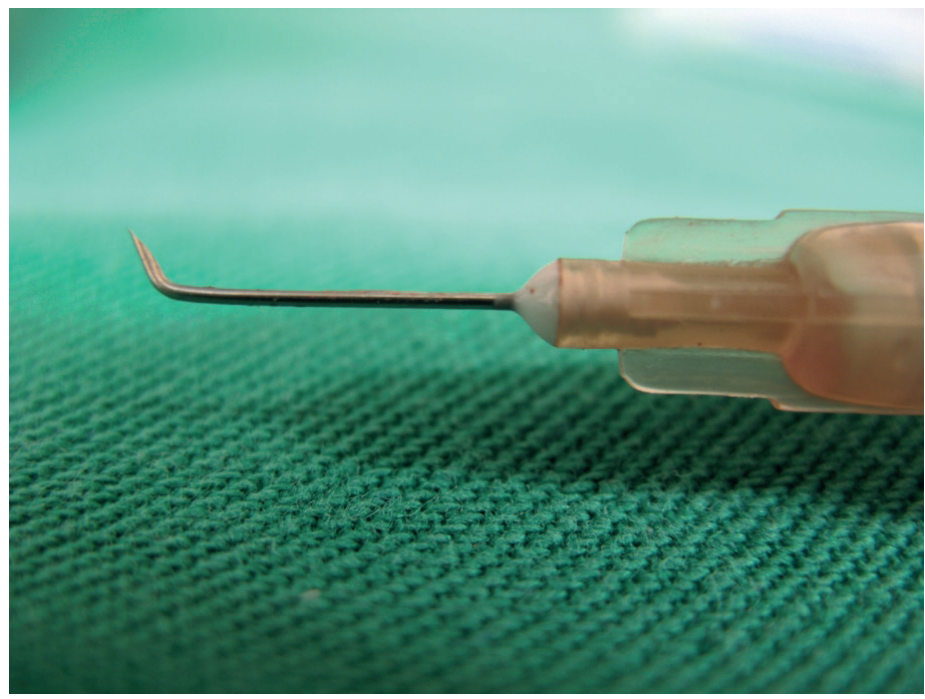

FIGURE 6 - Detail of the insulin needle. 


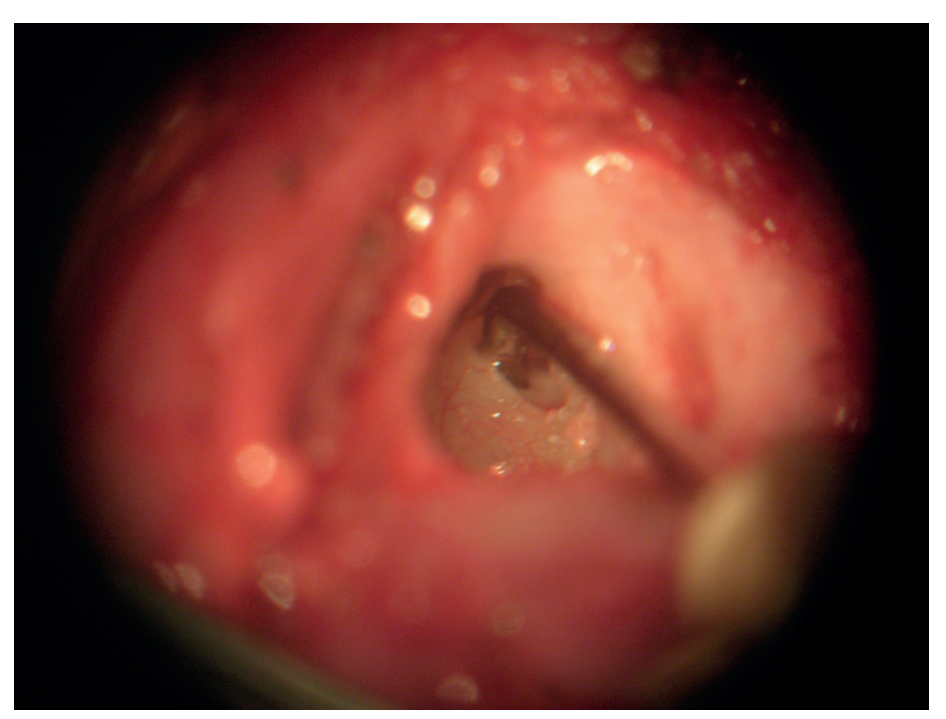

FIGURE 7 - Aspiration of cochlear fluid through the round window.

Nitric oxide in cochlear fluid was evaluated by the chemoluminescent method: Nitric Oxide Analyzer (NOA 280, Sievers Intruments, Inc, Boulder, CO, USA). This is a high sensible system to detect NO. Nitrite and nitrate present in these samples are reduced to NO by vanadium; and NO in its gas state is captured in a specific part of the device, and reacts with ozone resulting in a light emission, as the following reaction: $\mathrm{NO}+\mathrm{O}_{3} \rightarrow$ $\mathrm{NO}_{2}^{-}+\mathrm{O}_{2} / \mathrm{NO}_{2}^{-} \rightarrow \mathrm{NO}_{2}+\mathrm{hv}$

The light emission from a nitrogen dioxide electron from infrared spectrum region is detected by a photomultiplier tube. This lecture is done by NO Analysis (NOA) software and the results are obtained in $\mu \mathrm{M}$ of NO. The sensibility of NOA software to measure gas state $\mathrm{NO}$ is about 1 picomol.

A minimum of $10 \mu \mathrm{L}$ is needed for this measurement which is exactly the maximum volume that is obtained from the internal ear of these guinea pigs. Therefore, fluid from both ears was used to have this dosage performed.

\section{Results}

The final volume of cochlear fluid collected from each ear varied between 5 and $6 \mu \mathrm{M}$. At least $10 \mu \mathrm{M}$ of fluid was needed to perform a reliable NOA analysis, therefore fluids from both ears were collected to have a satisfactory NO count. As the estimated volume of cochlear fluids (endolymph and perilymph) in guinea pig is $8.5 \mu \mathrm{M}^{12}$ in this experimental work the volume of cochlear fluid collected was about 60 to $70 \%$ of the total volume of the cochlear fluids.

NOA showed an average of $12.55 \mu \mathrm{M}$ of nitric oxide in cochlear fluids of guinea pigs (Table 1).
TABLE 1 - Nitric oxide levels in guinea pigs cochlear fluids.

\begin{tabular}{ccc} 
Animals & Nitic Oxide $(\mu \mathrm{M})$ & Average $(\mu \mathrm{M})$ \\
\hline Guinea Pig I & 8.0 & \\
Guinea Pig II & 14.5 & 12.55 \\
Guinea Pig III & 13.27 & \\
Guinea Pig IV & 14.0 & \\
\hline
\end{tabular}

\section{Discussion}

Experimental models that can simulate tissue and cellular complex physiological mechanisms, enabling the evaluation of the molecular activities of these structures, constitute currently the paradigms of the experimental models.

They are very important to scientific research, because through them it is possible to control multiple variables and a wide range of studies can be done. Studies about efficiency and toxicity of medicines, physiopathology of certain conditions, follow-up of surgical intervention, are the main applications of these models ${ }^{13}$.

In Otology, many researchers developed experimental studies using guinea pigs (Cavia porcellus). This animal is preferably used due to the fact that its anatomy favors inner and medium ear surgical procedures. Wysocki ${ }^{2}$ in a recent work has described the guinea pig's anatomy in details facilitating the study of an access to the structures of the cochlear vestibular system.

Many inner ear diseases such as ischemia, acoustic trauma and ototoxic medicine damage may cause irreversible $e^{3,5,8}$. NO role related to cellular injury process is being the target of countless studies. Production of NO in the inner ear is well documented, and it is well-known that the physiological amount of this free radical mean no harm. Lesion occurs when there is an $\mathrm{NO}$ overproduction that is not followed by an increase in anti free radicals ${ }^{10}$.

NO measurement in experimental studies constitutes a challenge because of its high volatility. Most of the studies measure its stable metabolites such as the nitrite and nitrate, or their conversion enzymes. An example of this statement is the work done by Ruan et al. ${ }^{15}$ studying NO action in normal and ischemic cochlea of guinea pigs. They have used donors of NO such as S-nitrocysteine and nitroglycerin, as well as NO inhibitors such as N-nitro-L-arginine methyl ester. These authors infused either the NO donor or its inhibitor in the perilymphatic space through two little openings made in the basal gyrus of the cochlea, the remainder perilymph was drained by an additional opening. Guinea pigs were sacrificed two days later and cochleae were 
taken for study.

Some of them were artificially induced into the state of hypoxia and others were submitted to NO count. Hypoxia was performed in a closed environment without oxygen by exposing the cochlea for 20 to 120 minutes. Measurement was done using Griess colorimetric assay. Perylymph nitrite and nitrate were mixed to Griess reagent and their absorbance was registered in a $540 \mathrm{~nm}$ wave spectrophotometer. Absorbance was compared to a pre established standard curve that showed $\mathrm{NO}$ values for the sample $^{15}$. It is an indirect method to measure NO and the authors showed that in ischemic cochlea there is a NO decrease and the administration of exogenous NO could protect guinea pigs inner ear.

NO can also be measured by using its conversion enzyme as cited by Heinrich et al. ${ }^{16}$. They described a NO increase after a transtympanic injection of gentamicin in guinea pig cochlea. After five days of drug administration animals were sacrificed and cochleae were prepared for histological and immunochemistry evaluation using anti-eNOS antibody. Gentamicin treated animals had a high level of cochlear injury together with an increase in conversion enzyme eNOS ${ }^{16}$. The advantage of this method is the possibility of distinguishing which conversion enzyme was increased; it is known that in normal conditions there is no inducible form of NOS in the cochlea, an anti iNOS antibody can be used to identify any increase in NO levels in the inner ear.

Takumida et al. ${ }^{17}$ published the first work of 'NO direct measurement', using a substance called 4.5-diaminofluorescein diacetate (DAF-2DA). This substance is able to cross the plasmatic cells membrane and once in the cytoplasm, it is hydrolized into a new compound called 4.5-diaminofluorescein (DAF-2). DAF-2 reacts with the existing intracellular $\mathrm{NO}$, giving rise to triazolfluorescein (DAF-2T), that is a fluorescent green indicator, which is brighter, as it is higher is the intracellular NO.

In our study the chemoluminescent method was used to measure NO levels, since it is more sensitive to the slightest variation of NO quantity. This technique was used by Chen et $a l .{ }^{19}$ to demonstrate that the effects caused by hearing trauma can produce NO in the lateral wall of the cochlea. Guinea pigs were submitted to 40 hours of $105 \mathrm{dBSPL}$ acoustic trauma, and their hearing was then evaluated by means of short latency evoked potential after zero, seven, 28 and 56 days. Following the last evaluation the animals were sacrificed, the cochlea removed and the otic capsule exposed. Lateral wall, stria vascularis and spiral ligament were removed by micro dissection, homogenized, and centrifuged. The supernatant was then analyzed by NOA. NO levels were increased after exposition to noise, and lingered for 28 days, decreasing to normal levels after 56 days. Hearing evaluation showed an increase of the hearing thresholds during the first days, decreasing after some days, but normal levels were not reached ${ }^{18,19}$.

There was no reference in the literature about the direct measurement of NO in aspirated cochlear liquids. The term "cochlear fluids" are here preferentially used because even though the approach being done by the round window. We believe that the negative pressure given by the micropipette and the manipulation of the needle would lead to a rupture of the tender membranes of the membranaceous labyrinth and the final aspirated product is probably a mixture of endo and perilymph.

The technique used in this study to obtain cochlear fluids was efficient, and enabled the NO concentration measurement. Our technique stands out due to its feasibility and its application to many other studies such as ototoxicity, hearing trauma, and others. Furthermore, its cost is low enough to facilitate research in this area, and it does not need special dyes or fixatives.

The only one difficulty found was the small amount of cochlear fluids obtained by aspiration, collection from both cochleae were needed so as to have NO measured. We believe that can be improve together with the improvement of aspiration technique.

\section{Conclusions}

This study demonstrates the feasibility of collecting cochlear fluids in vivo and the possibility to dose nitric oxide level. This cost effective experimental model may contribute to future studies dealing with nitric oxide and inner ear.

\section{References}

1. Goksu N, Haziroglu R, Kemaloglu Y, Karademir N, Bayramoglu, I, Akyildiz N. Anatomy of the guinea pig temporal bone. Ann Otol Rhinol Laryngol. 1992;101(8): 699-704.

2. Wysocki J. Topographical anatomy of the guinea pig temporal bone. Hear Res. 2005;199(1-2):103-10.

3. Morizane I, Hakuba N, Hyodo J, Shimizu Y, Fujita K, Yoshida $\mathrm{T}$, Gyo K. Ischemic damage increases nitric oxide production via inducible nitric oxide synthase in the cochlea. Neurosci Lett. 2005;391(1-2):62-7.

4. Nakagawa T, Yamane H, Takayama M, Sunami K, Nakai Y. Involvement of nitric oxide in aminoglycoside vestibulotoxicity in guinea pigs. Neurosci Lett. 1999;267(1):57-60.

5. Watanabe K, Inai S, Hess A, Michel O, Yagi T. Acoustic stimulation promotes the expression of inducible nitric oxide synthase in the vestibule of guinea pigs. Acta Otolaryngol Suppl. 2004;(553):54-7.

6. Palmer RM, Ashton DS, Moncada S. Vascular endothelial cells synthesize nitric oxide from L-arginine. Nature. 1988;333(6174):6646. 
7. Bredt DS, Hwang PM, Glatt CE, Lowenstein C, Reed RR, Snyder SH. Cloned and expressed nitric oxide synthase structurally resembles cytochrome P-450 reductase. Nature. 1991;351(6329):714-8.

8. Förstermann U, Pollock JS, Schmidt HH, Heller M, Murad F. Calmodulin-dependent endothelium-derived relaxing factor/nitric oxide synthase activity is present in the particulate and cytosolic fractions of bovine aortic endothelial cells. Proc Natl Acad Sci USA 1991;88(5):1788-92.

9. Fessenden JD, Coling DE, Schacht J. Detection and characterization of nitric oxide synthase in the mammalian cochlea. Brain Res. 1994;668(1-2):9-15.

10. Fessenden JD, Schacht J. The nitric oxide/cyclic GMP pathway: a potential major regulator of cochlear physiology. Hear Res. 1998;118(1-2):168-76.

11. Jero J, Coling DE, Lalwani AK. The use of Preyer's reflex in evaluation of hearing in mice. Acta Otolaryngol. 2001;121(5):5859.

12. Shinomori Y, Spack DS, Jones DD, Kimura RS. Volumetric and dimensional analysis of the guinea pig inner ear. Ann Otol Rhinol Laryngol. 2001;110(1):91-8.

13. Ferreira LM, Hochman B, Barbosa MV. Modelos experimentais em pesquisa. Acta Cir. Bras. 2005;20(Suppl 2):28-34.

14. Heinrich UR, Helling K, Sifferath M, Brieger J, Li H, Schmidtmann I, Mann WJ. Gentamicin increases nitric oxide production and induces hearing loss in guinea pigs. Laryngoscope. 2008;118(8):1438-42.

15. Ruan RS, Leong SK, Yeoh KH. Effects of nitric oxide on normal and ischemic cochlea of the guinea pig. Exp Neurol. 2001;169(1):200-7.

16. Heinrich UR, Selivanova O, Brieger J, Mann WJ. Endothelial nitric oxide synthase upregulation in the cochlea of the guinea pig after intratympanic gentamicin injection. Eur Arch Otorhinolaryngol. 2006;263(1):62-8

17. Takumida M, Anniko M. Direct evidence of nitric oxide production in guinea pig vestibular sensory cells. Acta Otolaryngol. 2000;120(1):34-8.

18. Chen YS, Tseng FY, Liu TC, Lin-Shiau SY, Hsu CJ. Involvement of nitric oxide generation in noise-induced temporary threshold shift in guinea pigs. Hear Res. 2005;203(1-2):94-100.

19. Chen YS, Tseng FY, Lin KN, Yang TH, Lin-Shiau SY, Hsu CJ. Chronologic changes of nitric oxide concentration in the cochlear lateral wall and its role in noise-induced permanent threshold shift. Laryngoscope. 2008;118(5):832-6.

\section{Correspondence:}

Fernando Kaoru Yonamine

Rua Bueno de Andrade, 706

01526-000 São Paulo - SP Brasil

Tel.: (5511)2671-2969/98315-6179

ferky@uol.com.br

Received: January 13, 2013

Review: March 17, 2013

Accepted: April 14, 2013

Conflict of interest: none

Financial source: none

${ }^{1}$ Research performed at Department of Otorhinolaryngology, Head and Neck Surgery and Division of Nephrology, Federal University of Sao Paulo (UNIFESP), Brazil. 